\title{
The prognostic value of phosphorylated Akt in breast cancer: a systematic review
}

SUBJECT AREAS:

PROGNOSTIC MARKERS

BREAST CANCER

Received

8 August 2014

Accepted

11 December 2014

Published

13 January 2015

Correspondence and requests for materials should be addressed to C.M. (maochen@cuhk. edu.hk) or J.-L.T. (jltang@cuhk.edu.hk)

* These authors contributed equally to this work.

\author{
Zu-Yao Yang ${ }^{1 *}$, Meng-Yang Di ${ }^{1 *}$, Jin-Qiu Yuan', Wei-Xi Shen², Da-Yong Zheng ${ }^{3}$, Jin-Zhang Chen ${ }^{3}$, \\ Chen $\mathrm{Mao}^{1,4}$ \& Jin-Ling Tang ${ }^{1,4,5}$
}

The prognostic value of phosphorylated Akt (pAkt) overexpression in breast cancer has been investigated by many studies with inconsistent results. This systematic review was conducted to evaluate the association of pAkt overexpression with breast cancer prognosis in terms of overall survival and disease-free survival. Three electronic databases (PubMed, EMBASE and Chinese Biomedical Literature Database) were comprehensively searched. Hazard ratios (HRs) with $95 \%$ confidence intervals (CIs) from different studies were combined using the random-effects model. In total, 33 studies with 9,836 patients were included for final analysis. The summary HR for overall survival and disease-free survival was 1.52 (95\% CI: 1.29-1.78) and 1.28 (95\% CI: 1.13-1.45), respectively, indicating higher risk of death and disease recurrence associated with pAkt overexpression. The results were robust in sensitivity analyses by omitting one study each time and by using the fixed-effects model instead. Subgroup and meta-regression analyses did not show that the prognostic effect of pAkt overexpression would change materially with such factors as population, status of hormone receptors, hormonal or trastuzumab treatment given, analyzing method (univariate versus multivariate) and methodological quality of the original studies. In conclusion, the available evidence suggests that pAkt overexpression is an adverse prognostic factor for breast cancer.
$\mathrm{B}$ reast cancer has long been the most frequent cancer among women worldwide, with an estimated 1.67 million new cases diagnosed each year (25\% of all cancers) $)^{1}$. Despite the significant progress in early detection and treatment over the past decades, breast cancer remains the leading cause of cancer deaths in women in many countries especially the less developed ones ${ }^{1}$. To achieve better management of breast cancer, the identification of clinical, pathological and biological factors that have prognostic value is very important, as those factors could be used to inform risk stratification, treatment selection and development of new therapeutic strategies ${ }^{2}$. Examples of such factors include tumor size, lymph node status, estrogen receptor (ER) status and human epidermal growth factor receptor 2 (HER2) status, which have been well integrated into clinical practice and contributed much to the improvement of breast cancer prognosis. Along with the emphasis on personalized medicine in recent years, increasing attention has been drawn to other biomarkers that may help explain residual risk not accounted for by the aforementioned traditional factors ${ }^{2}$.

Akt, also known as protein kinase $B$, is a serine/threonine protein kinase that, once activated by phosphorylation at serine 473 and threonine 308 , plays an important role in multiple cellular processes ${ }^{3}$. In particular, phosphorylated Akt (pAkt) may induce signals interfering with the apoptotic functions of the cell, and promote cell survival, proliferation and motility possibly through activation of mammalian target of rapamycin among other mechanisms ${ }^{3-6}$. Overexpressed pAkt is frequently observed in human lung, gastric, hepatocellular, pancreatic, renal, prostate and endometrial cancer as well as multiple myeloma ${ }^{7-11}$. Studies have documented the prognostic role of pAkt overexpression in some cancers. For example, a recent meta-analysis showed that pAkt overexpression was significantly associated with worse overall survival in non-small cell lung cancer patients (hazard ratio [HR]: 1.38, 95\% confidence interval [CI]: 1.11-1.70) ${ }^{12}$.

In breast cancer, the prognostic impact of this biomarker has also been evaluated by many studies, but their results were inconsistent. For example, the study of Xia et al with 130 patients found that pAkt overexpression was significantly associated with worse overall survival (HR: 2.16, 95\% CI: $1.22-3.81)^{13}$. However, in the study of Fabi et al with 73 patients, no significant association between pAkt status and overall survival was found $(P=0.97)^{14}$. 
The discrepancy between individual studies could have been due to multiple reasons such as different populations, sample sizes, methodological problems, and other potential confounding factors. Against this background, we conducted a comprehensive systematic review with an aim to clarify the prognostic value of pAkt overexpression in breast cancer. The potential impact of various factors on pAkt's prognostic effect was also investigated.

\section{Results}

Study selection and characteristics. The flow of study selection is shown in Figure 1. Initially, 2,976 records, including 1,063 duplicates, were identified by our literature search. Among the 1,913 unique records, 173 studies were subject to full text examination and 33 studies were considered eligible and finally included for the present systematic review ${ }^{6,13-44}$. Two studies ${ }^{33,34}$ were based on a same cohort with focus on different outcomes. The characteristics of the 33 studies are summarized in Table 1 . Their sample sizes ranged from 44 to 1,355 , with a median of 142 . In total, 9,836 patients were included for analysis. All studies assessed pAkt status by immunohistochemistry, and most of them used mouse anti-pAkt (Ser473) antibodies. pAkt overexpression was found in $12.7 \%$ to $87.5 \%$ of the subjects, with a summary rate of $49.3 \%$ (95\% CI: $42.4 \%-56.2 \%)$. Four and three studies clearly reported that all their

\section{6 references identified: 1057 PubMed \\ 1794 EMBASE (including conference abstracts) 125 Chinese Biomedical Literature Database \\ 1063 duplicates \\ 1913 abstracts screened}

1740 excluded:

163 editorials, letters, or reviews

1258 animal, in vivo or in vitro studies

63 not breast cancer patients

69 irrelevant or no biomarker

171 irrelevant outcomes

16 no detailed analysis on the prognostic value of biomarker

173 full texts further evaluated

140 excluded:

89 irrelevant biomarkers

6 irrelevant outcomes

43 no detailed analysis on the prognostic value of pAkt

2 duplicates

33 studies included

Figure $1 \mid$ Flow chart of study selection. subjects received trastuzumab and hormone treatment, respectively, while the other studies made no clear statement on this issue. The study quality scores based on the 9-point Newcastle-Ottawa scale ranged from 5 to 9 , with a median of 7 and a mean of 6.3.

Meta-analyses. HRs for overall survival were available from 20 of the 33 included studies (Table 1). Meta-analysis of the 20 studies with 6,349 patients showed that pAkt overexpression was significantly associated with worse overall survival in breast cancer. The summary HR was 1.52 (95\% CI: 1.29-1.78), with substantial heterogeneity among the studies $\left(I^{2}=58.4 \%, P=0.001\right)$ (Figure 2). Sensitivity analyses omitting one study each time showed that individually Wang 2009 and Wang C 2011 had the largest influence on the result. The summary HR became 1.46 (95\% CI: $1.26-1.71$; heterogeneity test $I^{2}=54.6 \%, P=0.002$ ) when Wang 2009 was omitted and 1.57 (95\% CI: 1.32-1.87; heterogeneity test $I^{2}=58.8 \%, P=0.001$ ) when Wang C 2011 was omitted. When the fixed-effects model was used instead of the random-effects model, the summary HR became 1.31 (95\% CI: 1.20-1.43).

HRs for disease-free survival were available from 24 of the 33 included studies (Table 1). Meta-analysis of the 24 studies with 8,683 patients showed that pAkt overexpression was significantly associated with worse disease-free survival in breast cancer. The summary HR was 1.28 (95\% CI: 1.13-1.45), with substantial heterogeneity among the studies $\left(I^{2}=74.2 \%, P<0.001\right)$ (Figure 3 ). Sensitivity analyses omitting one study each time showed that individually Aleskandarany 2011 and Yamamoto 2006 had the largest influence on the result. The summary HR became 1.33 (95\% CI: 1.15-1.53; heterogeneity test $\left.I^{2}=68.1 \%, P<0.001\right)$ when Aleskandarany 2011 was omitted and 1.24 (95\% CI: 1.10-1.40; heterogeneity test $I^{2}=72.2 \%, P<0.001$ ) when Yamamoto 2006 was omitted. When the fixed-effects model was used instead of the random-effects model, the summary HR became 1.06 (95\% CI: 1.021.10).

To investigate the heterogeneity detected in the above meta-analyses, a series of subgroup and meta-regression analyses were conducted as planned. Although the summary HRs were not statistically significant in some subgroups (e.g. the one with proportion of ERpositive patients $<50 \%$; the one in which trastuzumab was given to all patients), meta-regression analyses suggested that the betweensubgroup differences did not reach statistical significance (Table 2). More rigorous stratification of studies according to the stage of cancer, ER status, PR status, and HER2 status did not show significant difference between the subgroups either (for details, see Supplementary Table S1). Thus, there is no evidence to show that any of these factors could explain the heterogeneity. In other words, the prognostic effect of pAkt overexpression did not change materially with such factors as population, sample size, status of hormone receptors, and methodological features of the original studies.

Analysis of Publication Bias. The funnel plots corresponding to Figure 2 and Figure 3 demonstrated some degree of asymmetry (Egger's regression tests: $P<0.001$ and $P=0.009$, respectively) (Figure 4), which could be due to potential publication bias among other reasons ${ }^{45}$. After adjusting for the potential publication bias by trim-and-fill method, the summary HRs corresponding to Figure 2 and Figure 3 became 1.35 (95\% CI: 1.15-1.58) and 1.22 (95\% CI: 1.08-1.39), respectively. Although the adjusted estimates were slightly smaller than the unadjusted ones, they were still statistically significant and did not influence the original conclusion.

\section{Discussion}

The present systematic review included 33 studies with 9,836 patients to evaluate the prognostic effect of pAkt overexpression in breast cancer, representing the most comprehensive summary of available 


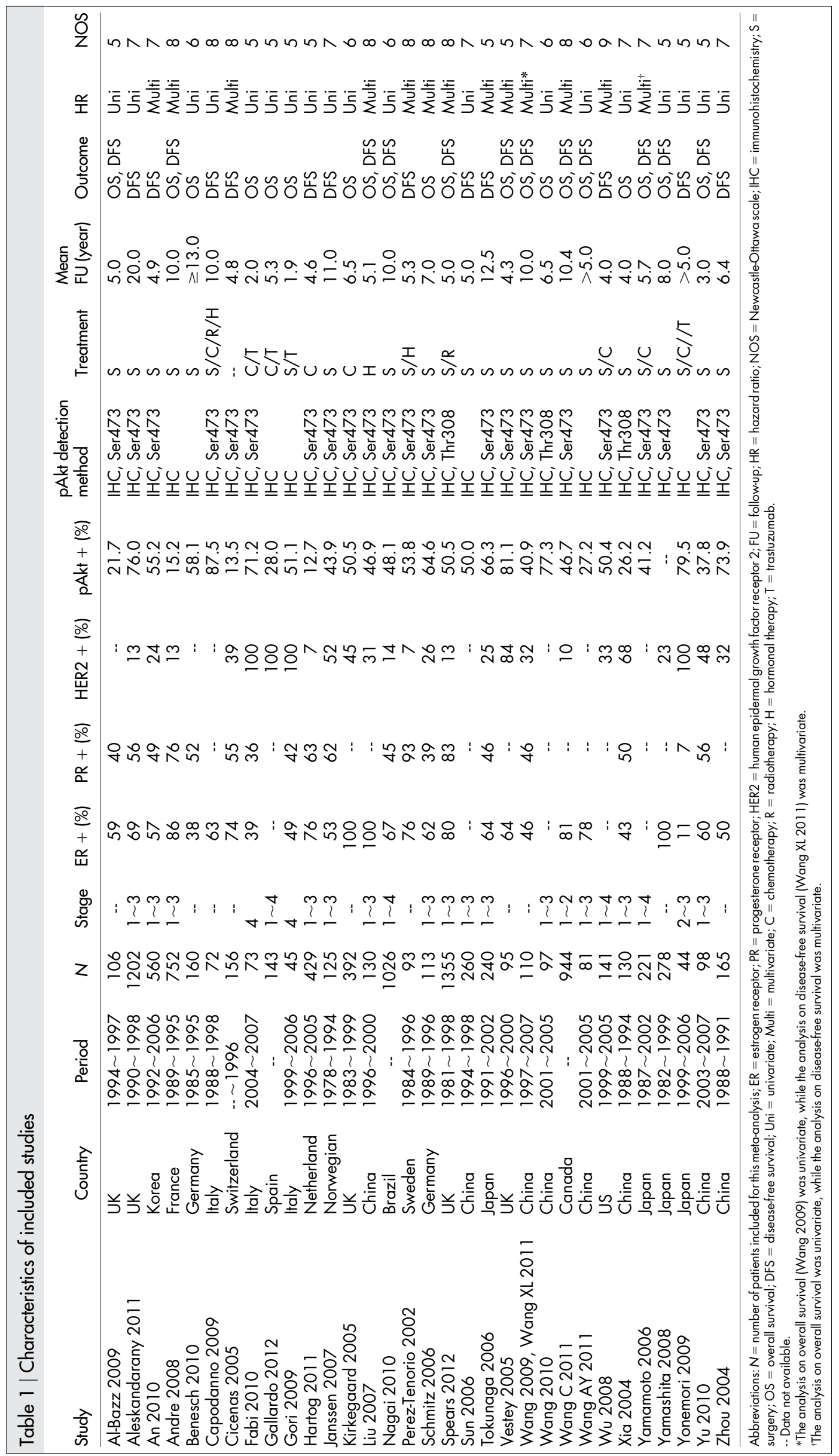




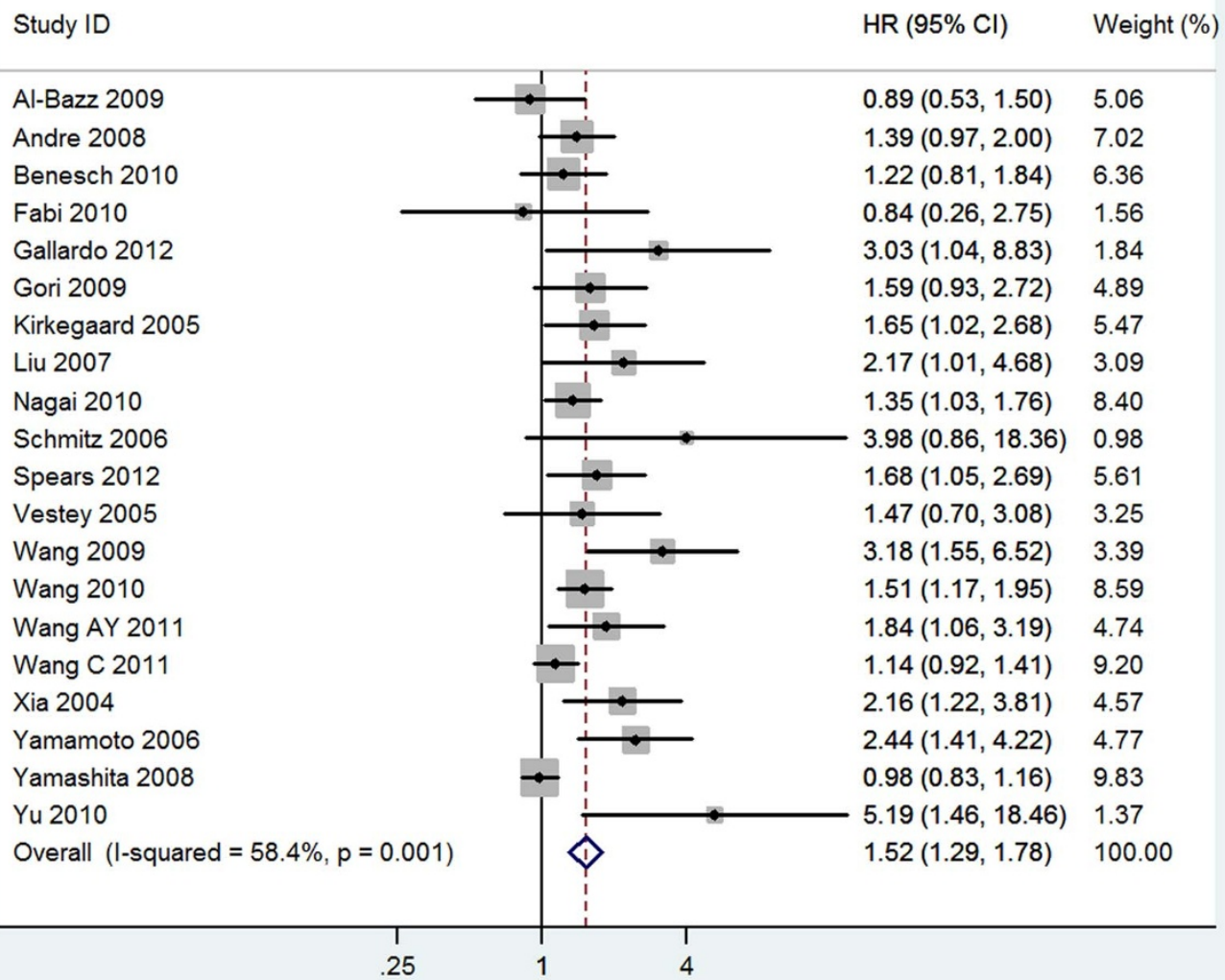

Figure $2 \mid$ Meta-analysis of the association between pAkt overexpression and overall survival in breast cancer. Results are presented as individual and pooled HRs with corresponding 95\% CIs. HR $>1$ means that overall survival of the patients with pAkt overexpression is worse than that of the pAktnegative ones, while $\mathrm{HR}<1$ means the opposite.

evidence on this topic so far. pAkt overexpression was found to be associated with both worse overall survival (HR: 1.52, 95\% CI: $1.29-$ 1.78 ) and worse disease-free survival (HR: $1.28,95 \%$ CI: 1.13-1.45) in breast cancer. Specifically, pAkt-overexpressed patients have a $50 \%$ higher risk of death and a $30 \%$ higher risk of disease recurrence compared with those without pAkt overexpression.

Substantial between-study heterogeneity was detected in our meta-analyses. However, subgroup and meta-regression analyses provided no evidence that any of the pre-specified factors such as population, status of hormone receptors, hormonal or trastuzumab treatment given, effect measure used in the original studies (HR vs. rate ratio), analyzing method (univariate vs multivariate) and study quality accounted for the heterogeneity. On one hand, this indicated that the prognostic effect of pAkt overexpression was robust in various scenarios, while on the other hand we can infer that there were other factors than the investigated ones existing as effect modifiers. Based on the data collected, we suggested that the varying scoring methods for pAkt status and definitions of pAkt overexpression have at least partly contributed to the between-study heterogeneity.

Specifically, we noted that the scoring methods used by published studies comprised of: (i) staining intensity alone; (ii) the proportion of tumor cells with positive staining alone; (iii) staining intensity score multiplied by or plus the score for the proportion of tumor cells with positive staining; or (iv) other derivative methods (for details, see Supplementary Table S2). With regard to each method, the cutoffs or thresholds used to define pAkt overexpression also varied. Due to the highly heterogeneous nature of these methods and definitions, we were unable to conduct meaningful subgroup or meta-regression analysis to investigate their impact on the observed prognostic effect of pAkt overexpression. This highlights the need for a standardized methodology for pAkt status testing before this biomarker can be applied to clinical practice.

The funnel plots corresponding to Figure 2 and Figure 3 were asymmetrical, which indicated that our meta-analyses might have suffered from publication bias. However, there were alternative explanations for this, as studies have shown that the asymmetry of funnel plots could be due to other reasons than publication bias, such as true heterogeneity of effects, poor study quality, and the play of chance $^{45}$. In view of the significant heterogeneity present in our meta-analyses, it is reasonable to say that publication bias possibly but not necessarily existed. Even if publication bias indeed occurred, our analysis showed that the summary HRs with publication bias adjusted for by trim-and-fill method were still statistically significant. Thus, we argue that publication bias did not constitute a major problem in the interpretation of our results.

Our finding about the adverse prognostic effect of pAkt overexpression is consistent with the observations in other solid tumors. For example, in the study of Nakanishi et al with 135 hepatocellular carcinoma patients, multivariate analysis identified pAkt overexpression as a strong predictor for early disease recurrence (relative risk: 12.5 , 95\% CI: 2.59-60.55) and poor prognosis (relative risk: 7.90, $95 \%$ CI: $1.25-50.00)^{9}$. The study of Cinti et al in 50 advanced gastric carcinomas showed that the five-year survival rate was $18 \%$ in the patients with pAkt overexpression versus $58 \%$ in the pAkt-negative ones $^{46}$. These findings together with ours suggest that pAkt overexpression could be a common prognostic factor shared by multiple types of human cancer, and thus it has the potential for being a therapeutic target of great clinical significance.

In fact, preclinical studies have provided evidence that inhibiting Akt activation while giving other treatments might enhance the overall efficacy. For example, Chen et al showed that inhibition of 


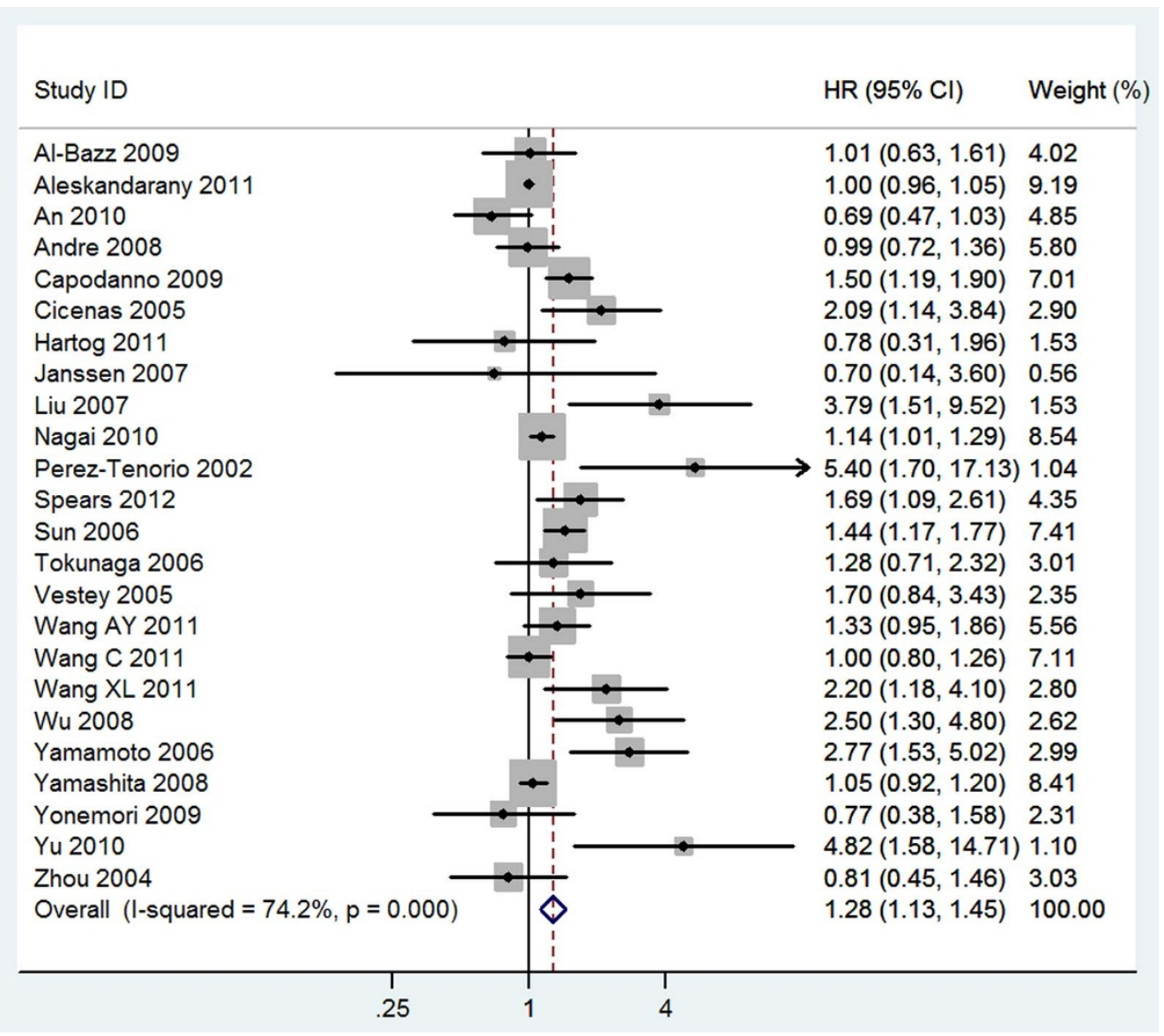

Figure 3 Meta-analysis of the association between pAkt overexpression and disease-free survival in breast cancer. Results are presented as individual and pooled HRs with corresponding 95\% CIs. HR $>1$ means that disease-free survival of the patients with pAkt overexpression is worse than that of the pAkt-negative ones, while HR $<1$ means the opposite.

Akt activation by recombinant VP1 suppresses the progression of hepatocellular carcinoma ${ }^{47}$. Other Akt inhibitors such as RX-0201, PBI-05204 and GSK2141795 have also demonstrated activity in various solid tumors in preclinical and phase I studies ${ }^{48}$. In breast cancer, in vitro and in vivo studies have showed that Akt inhibitor MK-2206, alone or in combination with chemotherapy, has antitumor activity and may augment the efficacy of existing cancer therapeutics ${ }^{49,50}$. Currently, MK-2206 is undergoing phase II trials ${ }^{51}$. Results of these studies should shed new lights on the clinical utility of pAkt testing. If the drugs targeted at pAkt proved effective, pAkt expression status could be a potential predictive biomarker and thus used to make the treatment of breast cancer more individualized in the future, similar to the role of EGFR mutation status in the EGFR-targeted treatment of non-small cell lung cancer ${ }^{52}$.

In conclusion, this systematic review suggests that pAkt overexpression is an adverse prognostic factor in breast cancer in terms of both overall survival and disease-free survival. To facilitate its application, efforts are needed to develop a standardized assay methodology and to further evaluate the efficacy of Akt inhibition with regard to other treatments in clinical settings.

\section{Methods}

Literature search. We performed a systematic search of PubMed, EMBASE (including the conference proceedings of American Society of Clinical Oncology and European Society of Medical Oncology) and Chinese Biomedical Literature Database (in Chinese) from their respective inception through 2013. The keywords used to search relevant publications included: "breast cancer*”, "breast carcinoma*”, "breast tumor*”, "breast tumour*”; “Akt*”, "pAkt”, "p-Akt"; "prognos*”, "outcome*”, "progress*”, "metasta*”, "relapse*”, "recurren*”, "surviv*”, "death*”, "die*”, "dead", "dying", "mortality". As the association of pAkt status with prognosis was often investigated by secondary analysis in the studies that focused on PTEN protein and/or PIK3CA gene, the following keywords related to the two biomarkers were also used in our literature search: "phosphatase and tensin homolog", "PTEN";

"PIK3CA", "PI3K*”, "PIK3*”, "phosphoinositide 3-kinase", "phosphoinositide-3kinase", "phosphatidylinositol 3 kinase", "phosphatidylinositol 3-kinase", "PI 3-

kinase", "phosphatidylinositol-3 kinase". No restrictions were placed on language or publication status. Wherever possible, the searches were limited to "human studies". The reference lists of eligible studies and relevant reviews were also scrutinized for additional eligible studies.

Study selection. The titles and abstracts of all identified records were screened to judge their relevance. The full texts of the studies seemingly fulfilling the inclusion criteria were obtained for further assessment. Cohort studies that met all of the following criteria were considered eligible: (i) The subjects were patients diagnosed with breast cancer. (ii) The outcomes included overall survival, disease-free survival, or both. (iii) pAkt status was tested and correlated with the outcomes. Duplicates and studies with non-extractable data were excluded.

Data extraction. The following data were extracted from eligible studies: (i) bibliographic information, such as first author, country and publication year; (ii) data on clinical and pathological characteristics of patients, such as sample size, stage of disease, ER status, PR status, HER2 status, and treatments given; (iii) the proportion of patients with pAkt overexpression; (iv) main results of the study, such as HR and $95 \%$ CI (if available, multivariate estimates were preferable); (v) the information related to study quality (see below).

Authors of the original studies were contacted as needed to clarify the ambiguities in the reported methods or results and to seek additional data not included in the published reports. If not explicitly reported in the original paper and still not available after contact with author, $\mathrm{HR}$ was estimated according to the survival curves using the method developed by Parmar et al and recommended by the Cochrane Handbook for Systematic Reviews ${ }^{53}$. In rare cases, $\mathrm{HR}$ was not estimable and rate ratio was used as a substitute for $\mathrm{it}^{54}$. Data extraction was completed independently by two reviewers (Z.Y.Y. \& M.Y.D.). Disagreements between the two were resolved by revisiting the original paper and discussion until consensus was reached.

Quality assessment. The quality of included studies was assessed according to the Newcastle-Ottawa scale ${ }^{55}$, which was frequently employed by previous studies ${ }^{56}$. This 


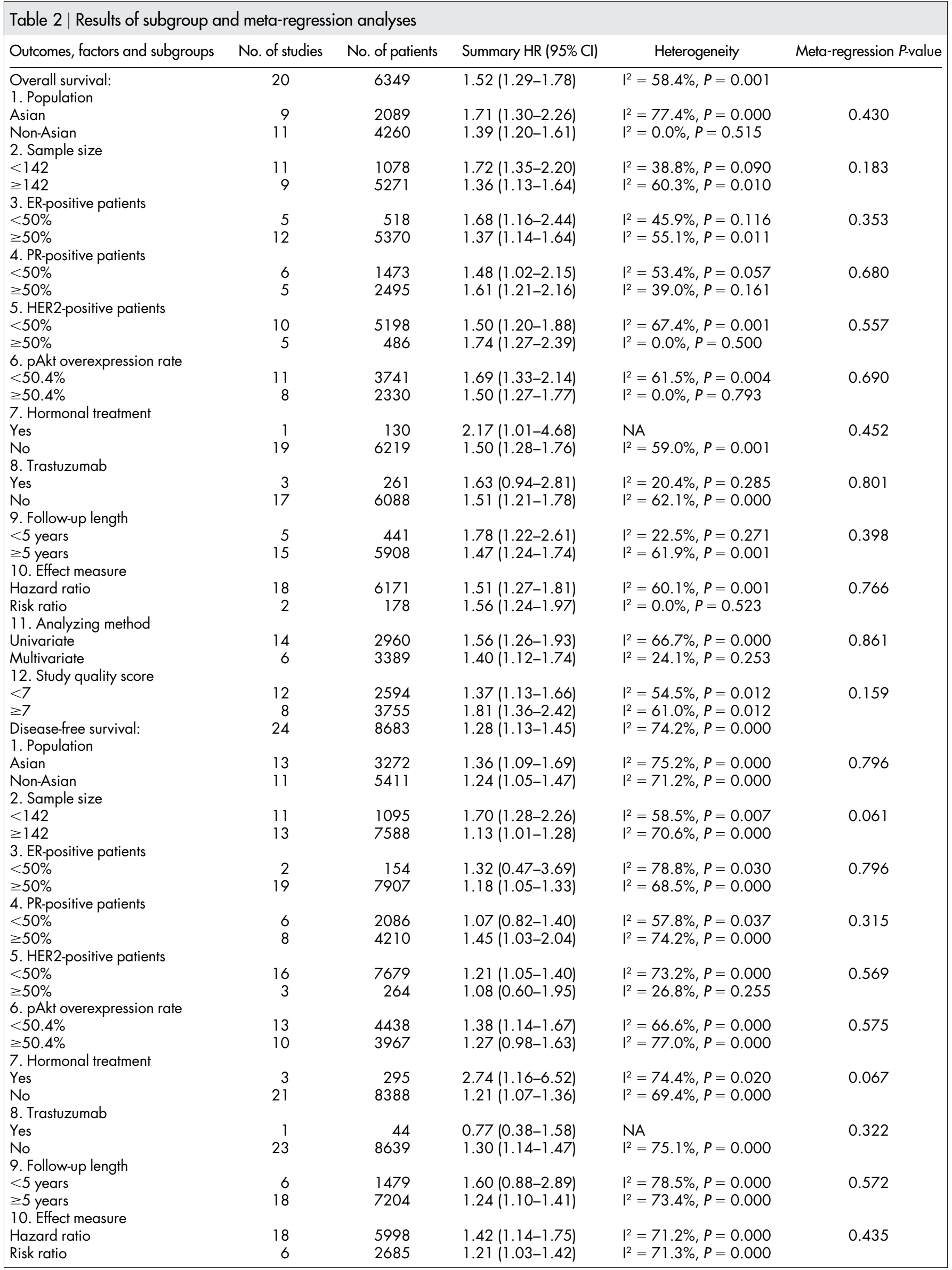




\section{Table 2 | Continued}

\begin{tabular}{lccccc} 
Outcomes, factors and subgroups & No. of studies & No. of patients & Summary HR $(95 \% \mathrm{Cl})$ & Heterogeneity & Meta-regression $P$-value \\
\hline $\begin{array}{l}11 \text {. Analyzing method } \\
\text { Univariate }\end{array}$ & 12 & 3886 & $1.16(1.02-1.31)$ & $\mathrm{I}^{2}=68.8 \%, P=0.000$ \\
$\begin{array}{l}\text { Multivariate } \\
\text { 12. Study quality score }\end{array}$ & 12 & 4797 & $1.65(1.22-2.22)$ & $\mathrm{I}^{2}=76.1 \%, P=0.000$ \\
$<7$ & 9 & 2397 & $1.14(1.00-1.31)$ & $\mathrm{I}^{2}=33.1 \%, P=0.153$ \\
$\geq 7$ & 15 & 6286 & $1.41(1.15-1.71)$ & $\mathrm{I}^{2}=81.4 \%, P=0.000$ \\
\hline Abbreviations: $\mathrm{HR}$ = hazard ratio; $\mathrm{Cl}=$ confidence interval; ER = estrogen receptor; PR = progesterone receptor; HER2 = human epi-dermal growth factor receptor $2 ; \mathrm{NA}=$ not applicable.
\end{tabular}

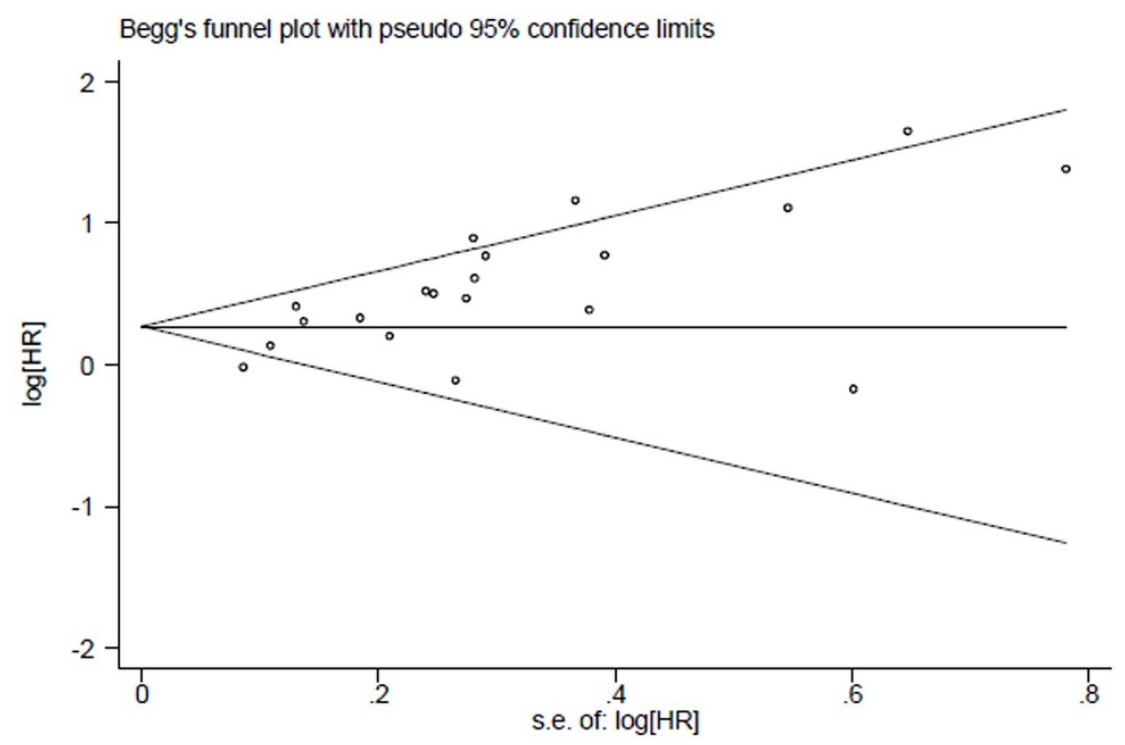

(A)

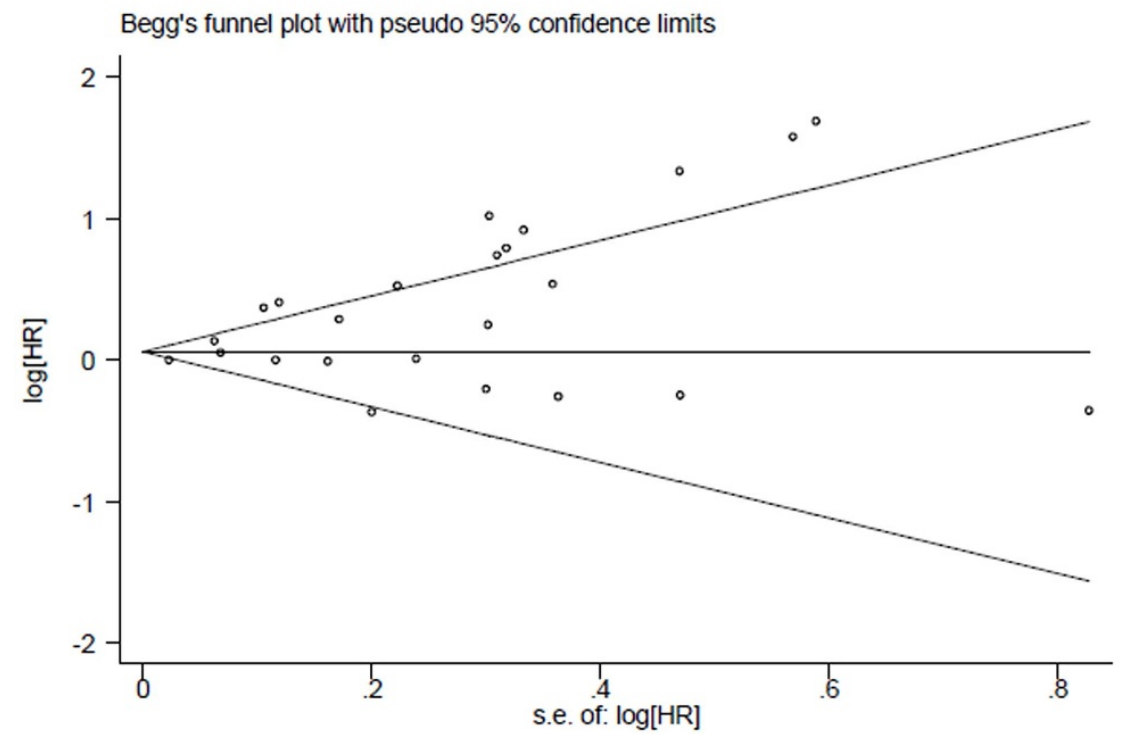

(B)

Figure $4 \mid$ Funnel plots to examine the possibility of publication bias in the data for overall survival (A) and that for disease-free survival (B). The standard error of log HR (S.E. of log HR) was plotted against log HR for each individual study as represented in a circle. Egger's tests showed that the funnel plots were asymmetric ( $P<0.001$ for Figure 4(A); $P=0.002$ for Figure $4(B))$, which could be due to potential publication bias among other reasons. 
scale focuses on three aspects of studies, including selection of patients, comparability of baseline characteristics, and outcome assessment. For each aspect, there are 1 4 items for detailed evaluation. The study quality was denoted by a numerical score ranging from 0 to 9 , with 9 representing the highest quality. Quality assessment was completed independently by two reviewers (J.Q.Y. \& C.M.). Disagreements between the two were resolved by revisiting the original paper and discussion. Unsettled disagreements were referred to a third researcher for final decision (J.L.T.).

Statistical analysis. The primary and secondary clinical outcomes of our interest were overall survival and disease-free survival, respectively. The effect of pAkt overexpression on the outcomes was measured by $\mathrm{HR}$ with $95 \% \mathrm{CI}$, and the HRs from relevant studies were combined to produce a summary HR for each outcome. HR $>1$ means that the prognosis of patients with overexpressed pAkt is worse than that of the other patients, while $\mathrm{HR}<1$ means the opposite. The statistical heterogeneity among studies was assessed by the Cochran's $Q$ test and the $I^{2}$ statistic $^{57,58}$. A $p$ value $\leq 0.10$ for the $Q$ test or an $I^{2}>50 \%$ was suggestive of substantial between-study heterogeneity. In case of substantial heterogeneity, the random-effects model was used for meta-analysis; otherwise, the fixed-effects model was used. If substantial, the heterogeneity was investigated by subgroup and meta-regression analyses to see if it could be explained by the following factors: population, sample size, the respective proportion of subjects positive for ER, PR and HER2, pAkt overexpression rate, hormonal treatment, trastuzumab treatment, length of follow-up, effect measure used in original studies (HR vs. rate ratio), analyzing method (univariate vs. multivariate) and study quality. Sensitivity analyses were conducted by omitting one study each time and by using the alternative analysis model (e.g. switching from the randomeffects model to the fixed-effects model). Begg's funnel plot and Egger's test were used to examine the possibility of publication bias if a meta-analysis included 10 or more studies $^{59}$. In presence of an asymmetric funnel plot, the Duval and Tweedie nonparametric trim-and-fill method was used to adjust for the potential publication bias and to obtain an adjusted summary HR from the corresponding meta-analysis ${ }^{60}$ All the analyses were performed with STATA software, version 11.0 (StataCorp, College Station, TX, USA).

1. International Agency for Research on Cancer. GLOBOCAN2012: estimated incidence, mortality and prevalence worldwide in 2012. http://globocan.iarc.fr/ Pages/fact_sheets_cancer.aspx [Accessed July 25, 2014].

2. Patani, N., Martin, L. A. \& Dowsett, M. Biomarkers for the clinical management of breast cancer: international perspective. Int J Cancer 133, 1-13 (2013).

3. Nicholson, K. M. \& Anderson, N. G. The protein kinase B/Akt signalling pathway in human malignancy. Cell Signal 14, 381-395 (2002).

4. Altomare, D. A. \& Testa, J. R. Perturbations of the AKT signaling pathway in human cancer. Oncogene 24, 7455-7464 (2005).

5. Xue, G. \& Hemmings, B. A. PKB/Akt-dependent regulation of cell motility. J Natl Cancer Inst 105, 393-404 (2013).

6. Al-Bazz, Y. O., Underwood, J. C., Brown, B. L. \& Dobson, P. R. Prognostic significance of Akt, phospho-Akt and BAD expression in primary breast cancer. Eur J Cancer 45, 694-704 (2009).

7. Alkan, S. \& Izban, K. F. Immunohistochemical localization of phosphorylated AKT in multiple myeloma. Blood 99, 2278-2279 (2002).

8. Cicenas J. The potential role of Akt phosphorylation in human cancers. Int J Biol Markers 23, 1-9 (2008)

9. Nakanishi, K., Sakamoto, M., Yamasaki, S., Todo, S. \& Hirohashi, S. Akt phosphorylation is a risk factor for early disease recurrence and poor prognosis in hepatocellular carcinoma. Cancer 103, 307-312 (2005).

10. Nam, S. Y. et al. Akt/PKB activation in gastric carcinomas correlates with clinicopathologic variables and prognosis. APMIS 111, 1105-1113 (2003).

11. Yang, X. et al. Significance of phosphoinositide 3 kinase/AKT pathway alterations in endometrial carcinoma [article in Chinese]. Zhonghua Bing Li Xue Za Zhi 40, 799-804 (2011).

12. Yang, Y. et al. Prognostic value of phospho-Akt in patients with non-small cell lung carcinoma: A meta-analysis. Int J Cancer 135, 1417-1424 (2014)

13. Xia, W. et al. Phosphorylation/cytoplasmic localization of p21Cip1/WAF1 is associated with HER2/neu overexpression and provides a novel combination predictor for poor prognosis in breast cancer patients. Clin Cancer Res 10, 3815-3824 (2004)

14. Fabi, A. et al. Clinical significance of PTEN and p-Akt co-expression in HER2positive metastatic breast cancer patients treated with trastuzumab-based therapies. Oncology 78, 141-149 (2010).

15. An, J. et al. Phosphorylated Akt and phosphorylated mTOR expression in breast invasive carcinomas: analysis of 530 cases. J Breast Cancer 13, 337-348 (2010).

16. Andre, F. et al. Expression patterns and predictive value of phosphorylated AKT in early-stage breast cancer. Ann Oncol 19, 315-320 (2008).

17. Benesch, C. et al. The clinicopathological and prognostic relevance of pyruvate kinase M2 and pAkt expression in breast cancer. Anticancer Res 30, 1689-1694 (2010).

18. Capodanno, A. et al. Dysregulated PI3K/Akt/PTEN pathway is a marker of a short disease-free survival in node-negative breast carcinoma. Hum Pathol $\mathbf{4 0}$ 1408-1417 (2009).

19. Cicenas, J. et al. Increased level of phosphorylated akt measured by chemiluminescence-linked immunosorbent assay is a predictor of poor prognosis in primary breast cancer overexpressing ErbB-2. Breast Cancer Res 7, R394-401 (2005).

20. Gallardo, A. et al. Increased signalling of EGFR and IGF1R, and deregulation of PTEN/PI3K/Akt pathway are related with trastuzumab resistance in HER2 breast carcinomas. Br J Cancer 106, 1367-1373 (2012).

21. Gori, S. et al. EGFR, pMAPK, pAkt and PTEN status by immunohistochemistry: correlation with clinical outcome in HER2-positive metastatic breast cancer patients treated with trastuzumab. Ann Oncol 20, 648-654 (2009).

22. Hartog, H. et al. Divergent effects of insulin-like growth factor-1 receptor expression on prognosis of estrogen receptor positive versus triple negative invasive ductal breast carcinoma. Breast Cancer Res Treat 129, 725-736 (2011).

23. Janssen, E. A. et al. Comparing the prognostic value of PTEN and Akt expression with the Mitotic Activity Index in adjuvant chemotherapy-treated node-negative breast cancer patients aged $<55$ years. Cell Oncol 29, 25-35 (2007).

24. Kirkegaard, T. et al. AKT activation predicts outcome in breast cancer patients treated with tamoxifen. J Pathol 207, 139-146 (2005).

25. Liu, C., Zhou, S., Ke, C. S., Li, N. P. \& Wu, R. L. Activation and prognostic significance of AKT, NF-kappaB and STAT3 in breast cancer with lymph node metastasis and estrogen receptor expression [article in Chinese]. Ai Zheng $\mathbf{2 6}$ 929-936 (2007)

26. Perez-Tenorio, G. \& Stal O. Activation of AKT/PKB in breast cancer predicts a worse outcome among endocrine treated patients. Br J Cancer 86, 540-545 (2002).

27. Schmitz, K. J. et al. Overexpression of cyclo-oxygenase- 2 is an independent predictor of unfavourable outcome in node-negative breast cancer, but is not associated with protein kinase B (Akt) and mitogen-activated protein kinase (ERK1/2, p38) activation or with Her-2/neu signalling pathways. JClin Pathol 59, 685-691 (2006)

28. Sun, L. M., Wang, J. L., Song, M. \& Song, J. Y. Activation and significance of Akt in signal regulatory pathway of human breast cancer [article in Chinese]. Zhonghua Zhong Liu Fang Zhi Za Zhi 13, 1232-1234 (2006).

29. Tokunaga, E. et al. Akt is frequently activated in HER $2 /$ neu-positive breast cancers and associated with poor prognosis among hormone-treated patients. Int $J$ Cancer 118, 284-289 (2006).

30. Vestey, S. B. et al. Activated Akt expression in breast cancer: correlation with p53, Hdm2 and patient outcome. Eur J Cancer 41, 1017-1025 (2005).

31. Wang, A. Y., Lin, X. Y., Wang, Q. X. \& Li, J. M. Expression and significance of pAkt, PTEN, and P-gp in patients with axillary node negative breast cancer [article in Chinese]. Shandong Yi Yao 51, 19-21 (2011).

32. Wang, L. X. \& Chen, L. R. Expression and significance of Galectin-3 and p-AKT in invasive ductal carcinoma [article in Chinese]. Shi Yong Zhong Liu Za Zhi 25, 638-641 (2010)

33. Wang, X. L. et al. Expression of Akt and its clinical significance in breast carcinoma [article in Chinese]. Lin Chuang Yu Shi Yan Bing Li Xue Za Zhi 25, 257-263 (2009).

34. Wang, X. L. et al. AKT signaling pathway in invasive ductal carcinoma of the breast: correlation with ERa, ER $\beta$ and HER-2 expression. Tumori 97, 185-190 (2011).

35. Yamamoto, O., Gomyo, Y., Hirooka, Y., Tsujitani, S. \& Ikeguchi, M. Expression of phospho-Akt and PTEN proteins in human breast cancer in relation to tumor progression and patient survival. Yonago Acta medica 49, 19-27 (2006).

36. Yamashita, H. et al. Low phosphorylation of estrogen receptor alpha (ERalpha) serine 118 and high phosphorylation of ERalpha serine 167 improve survival in ER-positive breast cancer. Endocr Relat Cancer 15, 755-763 (2008).

37. Yonemori, K. et al. Immunohistochemical expression of PTEN and phosphorylated Akt are not correlated with clinical outcome in breast cancer patients treated with trastuzumab-containing neo-adjuvant chemotherapy. Med Oncol 26, 344-349 (2009).

38. Yu, P., Zhou, L., Ke, W. \& Li, K. Clinical significance of pAKT and CD44v6 overexpression with breast cancer. J Cancer Res Clin Oncol 136, 1283-1292 (2010).

39. Zhou, X. et al. Activation of the Akt/mammalian target of rapamycin/4E-BP1 pathway by ErbB2 overexpression predicts tumor progression in breast cancers. Clin Cancer Res 10, 6779-6788 (2004).

40. Aleskandarany, M. A. et al. Clinicopathologic and molecular significance of phospho-Akt expression in early invasive breast cancer. Breast Cancer Res Treat 127, 407-416 (2011).

41. Nagai, M. A. et al. Down-regulation of the candidate tumor suppressor gene PAR4 is associated with poor prognosis in breast cancer. Int J Oncol 37, 41-49 (2010).

42. Spears, M. et al. Proximity ligation assays for isoform-specific Akt activation in breast cancer identify activated Akt1 as a driver of progression. J Pathol 227, 481-489 (2012).

43. Wang, C. et al. Expression of Abl interactor 1 and its prognostic significance in breast cancer: a tissue-array-based investigation. Breast Cancer Res Treat 129 , 373-386 (2011).

44. Wu, Y. et al. Clinical significance of Akt and HER2/neu overexpression in AfricanAmerican and Latina women with breast cancer. Breast Cancer Res 10, R3 (2008).

45. Tang, J. L. \& Liu, J. L. Misleading funnel plot for detection of bias in meta-analysis. J Clin Epidemiol 53, 477-484 (2000).

46. Cinti, C. et al. Activated Akt as an indicator of prognosis in gastric cancer. Virchows Arch 453, 449-455 (2008). 
47. Chen, T. A. et al. Recombinant VP1, an Akt inhibitor, suppresses progression of hepatocellular carcinoma by inducing apoptosis and modulation of CCL2 production. PLoS One 6, e23317 (2011).

48. Pal, S. K., Reckamp, K., Yu, H. \& Figlin, R. A. Akt inhibitors in clinical development for the treatment of cancer. Expert Opin Investig Drugs 19, 1355-1366 (2010).

49. Hirai, H. et al. MK-2206, an allosteric Akt inhibitor, enhances antitumor efficacy by standard chemotherapeutic agents or molecular targeted drugs in vitro and in vivo. Mol Cancer Ther 9, 1956-1967 (2010).

50. Sangai, T. et al. Biomarkers of response to Akt inhibitor MK-2206 in breast cancer. Clin Cancer Res 18, 5816-5828 (2012).

51. Funda, Meric-Bernstam. Akt Inhibitor MK2206 in Treating Patients With Advanced Breast Cancer. http://clinicaltrials.gov/show/NCT01277757 [Accessed July 25, 2014].

52. Lee, C. K. et al. Impact of EGFR inhibitor in non-small cell lung cancer on progression-free and overall survival: a meta-analysis. J Natl Cancer Inst $\mathbf{1 0 5}$, 595-605 (2013).

53. Parmar, M. K., Torri, V. \& Stewart, L. Extracting summary statistics to perform meta-analyses of the published literature for survival endpoints. Stat Med 17, 2815-2834 (1998).

54. Pan, A., Sun, Q., Okereke, O. I., Rexrode, K. M. \& Hu, F. B. Depression and risk of stroke morbidity and mortality: a meta-analysis and systematic review. JAMA 306, 1241-1249 (2011).

55. Wells, G. A. et al. The Newcastle-Ottawa Scale (NOS) for assessing the quality of non-randomised studies in meta-analyses. http://www.ohri.ca/programs/clinical_ epidemiology/oxford.asp [Accessed July 25, 2014].

56. Thosani, N. et al. Reduced risk of colorectal cancer with use of oral bisphosphonates: a systematic review and meta-analysis. J Clin Oncol 31, 623-630 (2013).

57. Deeks, J. J., Higgins, J. P. T. \& Altman, D. G. eds. Chapter 9: Analysing data and undertaking meta-analyses. In: Higgins, J. P. T. \& Green, S., eds. Cochrane Handbook for Systematic Reviews of Interventions. Version 5.1.0 [updated March 2011]. The Cochrane Collaboration, 2011. www.cochrane-handbook.org [Accessed July 25, 2014].
58. Higgins, J. P., Thompson, S. G., Deeks, J. J. \& Altman, D. G. Measuring inconsistency in meta-analyses. BMJ 327, 557-560 (2003).

59. Sterne, J. A. C., Egger, M. \& Moher, F. eds. Chapter 10: Addressing reporting biases. In: Higgins, J. P. T. \& Green, S. eds. Cochrane Handbook for Systematic Reviews of Interventions. Version 5.1.0 [updated March 2011]. The Cochrane Collaboration, 2011.www.cochrane-handbook.org [Accessed July 25, 2014].

60. Duval, S. \& Tweedie, R. Trim and fill: a simple funnelplot based method of testing and adjusting for publication bias in meta-analysis. Biometrics 56, 455-463 (2000).

\section{Author contributions}

Study concept and design: J.L.T., C.M. and Z.Y.Y. Acquisition of data: Z.Y.Y. and M.Y.D. Analysis and interpretation of data: Z.Y.Y., M.Y.D., J.L.T., C.M., J.Q.Y., W.X.S., D.Y.Z. and J.Z.C. Drafting of the manuscript: Z.Y.Y., M.Y.D. and J.Q.Y. Critical revision of the manuscript for important intellectual content: J.L.T., C.M., Z.Y.Y., M.Y.D., J.Q.Y., W.X.S., D.Y.Z. and J.Z.C. Study supervision: J.L.T. and C.M. All authors reviewed the manuscript.

\section{Additional information}

Supplementary information accompanies this paper at http://www.nature.com/ scientificreports

Competing financial interests: The authors declare no competing financial interests How to cite this article: Yang, Z.-Y. et al. The prognostic value of phosphorylated Akt in breast cancer: a systematic review. Sci. Rep. 5, 7758; DOI:10.1038/srep07758 (2015).

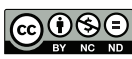

This work is licensed under a Creative Commons Attribution-NonCommercialNoDerivs 4.0 International License. The images or other third party material in this article are included in the article's Creative Commons license, unless indicated otherwise in the credit line; if the material is not included under the Creative Commons license, users will need to obtain permission from the license holder in order to reproduce the material. To view a copy of this license, visit http:// creativecommons.org/licenses/by-nc-nd/4.0/ 